\title{
Molecular Mechanisms of Root Development in Rice
}

\author{
Funing Meng ${ }^{\dagger}$, Dan Xiang ${ }^{\dagger}$, Jianshu Zhu, Yong Li and Chuanzao Mao ${ }^{*}$ (D)
}

\begin{abstract}
Roots are fundamentally important for growth and development, anchoring the plant to its growth substrate, facilitating water and nutrient uptake from the soil, and sensing and responding to environmental signals such as biotic and abiotic stresses. Understanding the molecular mechanisms controlling root architecture is essential for improving nutrient uptake efficiency and crop yields. In this review, we describe the progress being made in the identification of genes and regulatory pathways involved in the development of root systems in rice (Oryza sativa L.), including crown roots, lateral roots, root hairs, and root length. Genes involved in the adaptation of roots to the environmental nutrient status are reviewed, and strategies for further study and agricultural applications are discussed. The growth and development of rice roots are controlled by both genetic factors and environmental cues. Plant hormones, especially auxin and cytokinin, play important roles in root growth and development. Understanding the molecular mechanisms regulating root architecture and response to environmental signals can contribute to the genetic improvement of crop root systems, enhancing their adaptation to stressful environmental conditions.
\end{abstract}

Keywords: Rice, Root system architecture, Molecular mechanism, Genetic control

\section{Background}

A major challenge for plants is the complexity of the environment in which they must survive. Root systems are vital for addressing this complexity. Roots not only provide structural support to the aerial organs of the plant, and enable the acquisition of water and nutrients that are required for plant growth, but also can monitor environmental conditions in the soil, such as the water content, nutrient levels, and the presence of toxic elements. To ensure an optimal response to changing environmental situations, root systems are continuously reshaped by the initiation and elongation of new roots throughout the growth period, facilitating the plant's adaptation to biotic and abiotic stresses (Fukai and Cooper 1995; Gowda et al. 2011). Understanding the mechanisms that control root patterning and identifying the genes responsible for post-embryonic root initiation could therefore enable breeders to improve crop tolerance to abiotic stresses (Coudert et al. 2010).

\footnotetext{
*Correspondence: mcz@zju.edu.cn

${ }^{\dagger}$ Funing Meng and Dan Xiang contributed equally to this work.

State Key Laboratory of Plant Physiology and Biochemistry, College of Life Sciences, Zhejiang University, Hangzhou 310058, China
}

There are two main types of root systems in plants, defined by their developmental origin and branching patterns: taproot systems and fibrous root systems. The taproot system occurs in dicot plants such as Arabidopsis thaliana, tomato (Solanum lycopersicum), and pea (Pisum sativa), while fibrous root systems occur in monocots such as rice (Oryza sativa), wheat (Triticum aestivum), and maize (Zea mays) (Atkinson et al. 2014). In taproot systems, the primary root is the main root, and branching consists of secondary, smaller lateral roots (LRs) and root hairs. A fibrous root system, by contrast, consists of a dense mass of adventitious roots (also called crown roots in cereals) that arise from the stem, which are distinct from the primary root, LRs, and root hairs (Coudert et al. 2010). Because the primary root (embryonic root) dies as the monocots age, the adventitious roots are the main root tissues in the fibrous root system of monocot plants.

Rice is a monocot model plant that provides a good experimental system for addressing the molecular mechanisms of constitutive and adaptive root branching and development in fibrous root systems (Gojon et al. 2009). The genetic regulatory network of root development in Arabidopsis is not suitable for understanding this process 
in rice which, unlike Arabidopsis, forms many adventitious roots. Deciphering the molecular regulatory network of root development in rice is therefore vital for enhancing our understanding of the genetic regulation of root development in the monocots more generally. Furthermore, a thorough knowledge of the key genes involved in rice root development will enable breeders to improve the root system and generate high-yield and nutrient-efficient cultivars using genetic manipulation or marker-assisted selection.

With the rapid development of functional genomics, significant progress has been made in elucidating the genetic control of root development in rice using mutant and quantitative trait loci (QTL) analyses. Here, we review the current progress in identifying genes regulating the development of the rice root system, including the primary root, crown root, lateral root, and root hairs. This research will improve our understanding of root system architecture (RSA) development and ultimately enable breeders to select for ideal root architectures promoting higher-yielding crops.

\section{Main Text}

\section{Genes Regulating Root Length}

Root length is an important component of root architecture that is essential to survival in complex soil conditions. A number of QTLs have been found to play major roles in regulating root growth and development in rice (Zhang et al. 2001; Toorchi et al. 2002, 2007; Obara et al. 2010; Wang et al. 2013; Li et al. 2015a), many of which were summarized in a previous review (Coudert et al. 2010); however, most of these QTLs have not been cloned. Recently, a QTL controlling root thickness and length, qRT9, was found to encode a basic helixloop-helix (bHLH) transcription factor, OsbHLH120. OsbHLH120 expression was strongly induced by polyethylene glycol, salt, and the drought-response hormone abscisic acid (ABA), suggesting an association with drought avoidance (Li et al. 2015a).

In addition to these QTLs, several genes regulating root length have been identified in mutant analyses. The rice UDP-N-acetylglucosamine biosynthesis-related gene OsG NA1 controls root elongation, as the loss-of-function mutant Osgna1 exhibited a temperature-sensitive defect in root elongation, with disrupted microtubules and cell shrinkage in the root elongation zone (Jiang et al. 2005). A putative mannosyl-oligosaccharide glucosidase (OsMOGS), required for $\mathrm{N}$-glycan maturation, was also found to regulate root elongation by affecting cell division and elongation (Wang et al. 2014a). Additionally, two sugar metabolism-related genes were also found to be involved in root development. OsDGL1 encodes a DOLICHYL DIPHOSPHOO LIGOSACCHARIDE-PROTEIN GLYCOSYLTRANSFE RASE $48 \mathrm{kDa}$ subunit precursor, which has conserved functions with the oligosaccharyltransferase complex found in all eukaryotes; mutations in this gene cause a defect in N-glycosylation in the root, resulting in shorter root cells, smaller root meristems, and root cell death (Qin et al. 2013). OsCYT-INV1, which encodes an alkaline/neutral invertase, also plays an important role in root elongation, as the Oscyt-inv1 mutant produces short roots. Oscyt-inv1 was found to accumulate sucrose and had reduced levels of hexose; however, its short-root phenotype could be rescued by exogenously supplying glucose (Jia et al. 2008). These studies suggest that genes involved in sugar metabolism and the sugar-based modification of proteins play important roles in root elongation.

Recent studies have demonstrated that root elongation is also associated with cell wall biosynthesis. OsGLU3 encodes a putative membrane-bound endo-1,4- $\beta$-glucanase, which is necessary for root elongation in rice. The Osglu3 mutant produced short roots with lower cellulose contents in its root cell walls, while the exogenous application of glucose suppressed these phenotypic defects (Zhang et al. 2012). Moreover, silencing the rice $\alpha$-expansin gene OsEXPANSION 8 (OsEXPA8), which encodes a cell walllocalized protein expressed predominantly in the root and shoot, resulted in a shorter primary root, fewer LRs, and short root hairs, supporting the hypothesis that expansins are involved in root growth by mediating cell wall loosening (Wang et al. 2014b).

Furthermore, genes involved in a range of physiological pathways have also been shown to regulate root length in rice. A T-DNA insertion mutant of a rice glutamic acid receptor-like gene, OsGLR3.1, was found to produce a short root, and further analysis revealed that this gene is essential for the maintenance of cell division and survival in the root apical meristem (RAM) in early seedlings (Li et al. 2006). OsGatB encodes a subunit of tRNA-dependent amidotransferase, an essential enzyme involved in Gln-tRNA ${ }^{\text {Gln }}$ biosynthesis in mitochondria, and may promote primary root growth by maintaining mitochondrial structure and function to facilitate cell division and elongation in the root tip (Qin et al. 2016). OsASL1, an argininosuccinate lyase that catalyses the final step of arginine biosynthesis, is also required for primary root elongation in rice, suggesting that a specific concentration of arginine is required for normal root growth in rice (Xia et al. 2014). OsELICITOR 5 (OsEL5), a membrane-anchored RING-H2-type ubiquitin E3 ligase, maintains cell viability after the initiation of root primordia (Koiwai et al. 2007). In addition, OsSPR1, a mitochondrial protein with an Armadillo-like repeat domain, is involved in post-embryonic root elongation and ion homeostasis (Jia et al. 2011), while OsMYB1, encoding an R2R3-type transcription factor, regulates primary root elongation in a phosphate-dependent manner (Gu et al. 2017). 
Certain phytohormone-related genes can also regulate rice primary root growth. The auxin transporter mutant Osaux1 had a longer primary root and shorter root hairs than the wild type (WT) when grown in hydroponic culture (Yu et al. 2015), while knock-out lines of OsAUXIN RESPONSE FACTOR 12 (OsARF12) had shorter primary roots (Qi et al. 2012). The microRNA miR393 influences auxin signalling to mediate primary root and adventitious root development by regulating its target genes, OsTIR1 and OsAFB2, the rice orthologs of the Arabidopsis auxin receptors TRANSPORT INHIBITOR RESPONSE 1 (TIR1) and AUXIN SIGNALING F-Box 2 (AFB2), which interact with OsIAA1, an AUXIN/INDOLE ACETIC ACID (AUX/ IAA) regulatory protein (Bian et al. 2012). Moreover, silencing the expression of DNA TOPOISOMERASE 1 (TOP1), an essential manipulator of DNA topology during RNA transcription and DNA replication, strongly reduced rice root elongation and gravitropism by mis-regulating auxin signalling and its associated transporters (Shafiq et al. 2017). Ethylene (ET) also appears to be involved in root development in rice. OsEIL1, a transcription factor involved in the ethylene signalling pathway, promotes rice root elongation (Mao et al. 2006). In addition, a recent study showed that the gain-of-function mutant Osethylene responsive factor 2 (Oserf2) formed a shorter root than the WT, while silencing OsERF2 leads to a long-root phenotype. Further experiments revealed that
OsERF2 is required for the root response to both $\mathrm{ABA}$ and ethylene signalling (Xiao et al. 2016). Another gene, $S H B$, which encodes an AP2/ERF transcription factor, affects gibberellic acid biosynthesis, as well as the elongation and proliferation of root meristem cells ( $\mathrm{Li}$ et al. 2015b). Additionally, recent studies revealed that strigolactones (SL) are required for the induction of root elongation by nitric oxide in response to nitrogen and phosphate deficiencies in rice (Sun et al. 2016). A salicylic acid biosynthesis-related gene, OsAIM1, is also required for root growth in rice, through promoting reactive oxygen species (ROS) accumulation (Xu et al. 2017).

Although about 20 genes required for root elongation have been identified, most function in different genetic pathways (Fig. 1); therefore, further research is still required to elucidate the molecular regulatory mechanisms of root elongation in rice.

\section{Molecular Mechanisms of Crown Root (Adventitious Root) Development}

Crown roots are one of the most important components of fibrous root systems, but are absent from taproot systems. In rice, the crown root primordium originates from the innermost ground meristem cells adjacent to the peripheral cylinder of vascular bundles in the stem (Itoh et al. 2005).

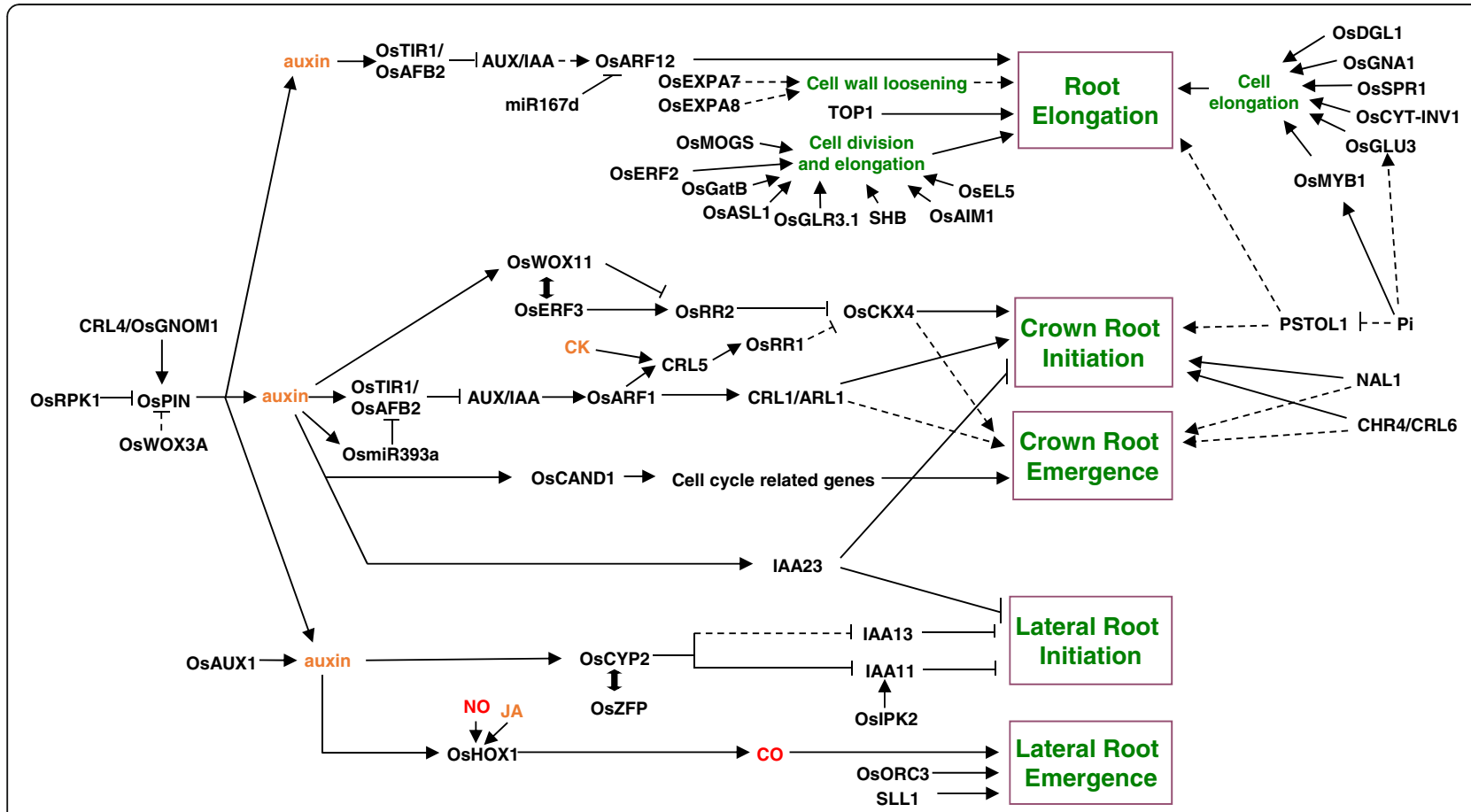

Fig. 1 The molecular regulatory mechanisms of root development in rice. Arrows represent positive regulatory actions. Lines ending in a flat head indicate a negative regulatory action. Dashed lines represent interactions that have not been experimentally confirmed. Double-headed arrows indicate that two proteins interact. Text color code: genes or protein, black; hormones, yellow; signals, red; biological processes, green 
The first crown root development gene to be identified was ADVENTITIOUS ROOTLESS 1/CROWN ROOTLESS 1 (ARL1/CRL1), encoding a LOB-domain transcription factor (Inukai et al. 2005; Liu et al. 2005); the knock-out mutant arl1, which carries a 20-bp deletion, produced no adventitious roots or adventitious root primordia throughout its entire lifespan (Liu et al. 2005), while the allelic line crll, containing a single amino acid change, occasionally produced crown roots at later developmental stages (Inukai et al. 2005). The shoots of the arl1/crl1 mutants are identical to the WT, however, while fewer LRs were observed on the primary root of the $\mathrm{crl} 1$ muant in comparison with the WT, the LR numbers on the primary root of arl1 were not significantly affected (Inukai et al. 2005; Liu et al. 2005). Furthermore, ARL1/CRL1 is a direct target of OsARF1, which can be induced by exogenous auxin treatment (Inukai et al. 2005). These results indicate that ARL1/CRL1 is a specific regulator of crown roots, with a central role in crown root initiation. Further research is required to elucidate how ARL1/CRL1 regulates crown root development.

Physiological studies have provided additional evidence of auxin playing an important role in crown root development, and the growth of functional genomics has meant that growing numbers of auxin-related genes have been found to be involved in this developmental process. Plants overexpressing the auxin biosynthesis gene OsYUCCA1 have a higher auxin content, which leads to more crown roots (Yamamoto et al. 2007). Two crown rootless mutants, crown rootless 4 (crl4) and Osgnom1, were found to be allelic lines with mutations in a membrane-associated guanine-nucleotide exchange factor of the ADP-ribosylation factor $G$ protein (GNOM). GNOM1 regulates the traffic of PIN-FORMED 1 (PIN1) auxin efflux carrier proteins, and consequently mediates polar auxin transport, suggesting that appropriate polarized auxin transport mediated by CRL4/OsGNOM1 is required for crown root initiation (Kitomi et al. 2008; Liu et al. 2009). Consistant with this, reducing the expression of OsPIN1 using RNA interference inhibited adventitious root development, a phenotype that could be rescued by the exogenous application of $\alpha$-naphthylacetic acid $(\alpha-N A A)$, suggesting a role for OsPIN1 in the regulation of adventitious root development via auxin pathway (Xu et al. 2005). Overexpressing OsRPK1, which encodes a leucine-rich repeat receptor-like kinase (LRRRLK), resulted in undeveloped adventitious roots and LRs, and a reduced RAM caused by lower expression of most OsPIN genes, suggesting that OsRPK1 also functions in an auxin-related pathway (Zou et al. 2014). Moreover, the gain-of-function mutant Osiaa23, which accumulates an auxin response protein, had defects in the initiation of its crown roots and LRs, and maintenance of the quiescent center (QC) in the root tip (Ni et al. 2011). Recently,
NARROW LEAF 1 (NAL1), was also found to mediate rice crown root development, as the loss-of-function mutant nal1 produced fewer adventitious roots. NAL1 encodes a putative trypsin-like serine/cysteine protease that affects the expression levels of many genes associated with leaf development and auxin transport; consequently, exogenous auxin treatment rescued the nal1 phenotype (Cho et al. 2014). Another auxin signalling gene, OsCAND1, named after its Arabidopsis homolog CULLIN-ASSOCIATED AND NEDDYLATION-DISSOCIATED 1 (CAND1), is also required for the emergence of crown roots (Wang et al. 2011). In addition, CHROMATIN REMODELING 4 (CHR4; Zhao et al. 2012), also named CROWN ROOTLESS 6 (CRL6; Wang et al. 2016), encodes a member of the large chromodomain, helicase/ATPase, and DNA-binding domain protein family, and is known to affect both auxin signalling and crown root development in rice. OsCHR4/CRL6 is most highly expressed in the basal region of the stem where crown roots are initiated. The defective crown root formation in crl6 can be rescued by auxin treatment, and furthermore, the expression of the OsIAA genes was down-regulated in crl6, providing evidence that $O s C H R 4 / C R L 6$ plays a role in crown root development through the auxin-signalling pathway (Wang et al. 2016).

Cytokinins (CK) are also essential for crown root development in rice. The WUSCHEL-related homeobox gene OsWOX11, expressed in emerging crown roots and cell division regions of the root meristem, is an important player in CK-regulated crown root development (Zhao et al. 2009, 2015b; Zhou et al. 2017). The loss of function or down-regulation of OsWOX11 results in a significant reduction in the number and elongation of crown roots, whereas overexpressing this gene significantly promotes crown root growth and dramatically increases root biomass. Further data showed that OsWOX11 directly represses $O s R R 2$, a type-A CK-responsive gene expressed in the crown root primordia, by binding to its promoter (Zhao et al. 2009). Moreover, OsERF3, an OsWOX11 interacting protein, positively regulates the expression of rice RESPONSE REGULATOR 2 (RR2) during crown root initiation; the OsERF3-OsWOX11 interaction likely represses OsRR2 expression during crown root elongation (Zhao et al. 2015b). OsWOX11 can also recruit the ADA2-GCN5 histone acetyltransferase to form a complex that commonly targets and regulates a set of root-specific genes involved in energy metabolism, cell wall biosynthesis, and the hormone response in the crown root meristem (Zhou et al. 2017). In addition, studies on another two genes, CRL5 and CK OXIDASE/DEHYDROGENASE 4 (OsCKX4), have provided further evidence that $C K$ signalling plays an important role in crown root emergence and development (Kitomi et al. 2011; Gao et al. 2014). CRL5 encodes an AP2/ERF transcription factor, expressed in the stem region 
where crown roots are initiated, and can be induced by exogenous auxin. The loss-of-function mutant $\mathrm{crl} 5$ produced fewer crown roots and its initiation of crown root primordia was impaired. Further data show that CRL5 positively regulates the type-A RR, OsRR1, to repress CK signalling, indicating that CRL5 integrates auxin and CK signalling to positively regulate crown root initiation in rice (Kitomi et al. 2011). OsCKX4, a CK oxidase/dehydrogenase (CKX) family gene, also plays a positive role in crown root formation; overexpressing OsCKX4 was found to increase the number of crown roots but reduce the overall height of the plant. OsCKX4 is a direct target of both the auxin response factor OsARF25 and the CK response regulators OsRR2 and OsRR3 (Gao et al. 2014). Consistant with this, overexpressing another RR gene, OsRR6, also suppressed root and vegetative development (Hirose et al. 2007). These data indicate that crown root development is co-ordinately regulated by auxin and CK signalling.

Increasingly, other plant hormones such as brassinosteroids (BRs) and SL have been implicated in crown root initiation and development. The brd1 mutant possesses a dysfunctional BR biosynthesis gene, OsBR6ox, and produces fewer crown roots (Mori et al. 2002). The dwarf (d) mutants with impaired SL biosynthesis and signalling produce fewer adventitious roots than the WT (Arite et al. 2012). Application of GR24, a synthetic SL analogue, increases the number of adventitious roots in the SL-deficient mutant $d 10$, but not in the SL-insensitive mutants $d 3$ and $d 14$, indicating that adventitious root production is positively regulated by SL in rice (Sun et al. 2015).

Though many genes involved in rice crown root development have been identified (Fig. 1), our knowledge of this process is still fragmented, and its molecular mechanisms still require substantial elucidation.

\section{Genes Controlling LR Development}

LRs are one of the most important root components, as they increase the root biomass, enabling the plant to absorb more water and nutrients as well as providing better anchorage in the soil. The molecular mechanisms regulating LR development are well studied in Arabidopsis, which revealed a major role for auxin signalling in this process (Benkova and Bielach 2010); however, LR development has not yet been well elucidated in rice, although significant progress has recently been made. Previously, two mutants, lateral rootless 1 (lrt1) and lrt2, were identified by their lack of LRs, and were found to be less sensitive to auxin (Chhun et al. 2003a; Wang et al. 2006; Faiyue et al. 2010). The altered lateral root formation 1 (alf1) mutant also formed significantly shorter LRs and was less sensitive to exogenous auxin than the WT (Debi et al. 2003). Additionally, two other auxin-resistant recessive mutants, auxin resistant mutant 1 (arm1) and $\operatorname{arm} 2$, failed to produce LRs (Chhun et al. 2003b).
These findings suggest that auxin signalling indeed participates in LR formation in rice; however, the corresponding genes have not been reported, with the exception of LRT2. Previous results indicate that OsCel9A, a rice glycoside hydrolase family gene, plays an essential role in regulating auxin-induced LR primordia formation (Yoshida et al. 2006). Additionally, NARROW LEAF 2 (NAL2) and NAL3, a pair of duplicated genes encoding WUSCHEL-RELATED HOMEOBOX 3A (OsWOX3A), also mediate LR development via the auxin signalling pathway. The nal2 nal3 double mutant showed a severe reduction in LR number and an increase in root hair number and length, and the expression of the OsPIN genes was also significantly affected (Cho et al. 2013). Consistant with this, two IAA family genes, OsIAA11 and OsIAA13, were reported to regulate LR formation, as LR development is inhibited in the gain-of-function mutants Osiaa11 and Osiaa13; however, the other root components, including the crown roots and root hairs, are not affected (Kitomi et al. 2012; Zhu et al. 2012). A recent study further indicates that the inositol polyphosphate kinase OsIPK2 interacts with OsIAA11 to protect it from degradation and thereby inhibits lateral root formation (Chen et al. 2017). Another IAA family gene, OsIAA23, is specifically expressed in the quiescent centre cells of the root tip during the development of primary, lateral, and crown roots, and the gain-of-function mutant Osiaa23 exhibited a pleiotropic phenotype, producing no crown root, no LRs, and no root cap (Ni et al. 2011).

Using a forward genetic approach, OsCYCLOPHILIN 2 (OsCYP2) was cloned and functionally identified, and the loss-of-function mutant Oscyp2 was found to exhibit defects in LR formation (Kang et al. 2013). Map-based cloning revealed that lrt 2 is allelic to Oscyp2 (Zheng et al. 2013). OsCYP2 is a cyclophilin-type peptidylprolyl cis/trans isomerase that efficiently catalyses the cis/ trans isomerization of OsIAA11 and directly regulates its stability; therefore, the OsCYP2 mutation reduces the interaction between OsTIR1 and OsIAA11, causing the accumulation of OsIAA11 and inhibiting auxin signalling-mediated LR development (Jing et al. 2015). A recent study demonstrated that OsZFP, a $\mathrm{C}_{2} \mathrm{HC}$-type zinc finger protein, can interact with OsCYP2 in the nucleus to regulate LR development (Cui et al. 2017). Furthermore, the inhibition of LR initiation was also reported in the auxin influx transporter mutant, Osaux 1 (Zhao et al. 2015a). These results indicate that auxin is a very important regulator of LR development in rice.

Other plant hormone signalling pathways have also been reported to influence LR growth and development; for example, HEME OXYGENASE 1 (HOX1) is regulated by jasmonic acid (JA) to control the formation of LRs through the production of carbon monoxide (Chen et al. 
2012; Hsu et al. 2012). Transgenic plants overexpressing DROUGHT STRESS RESPONSE-1 (OsDSR-1), the Arabidopsis ortholog of which encodes a putative calcium-binding protein, produce much shorter LRs when grown in media containing ABA, suggesting that OsDSR-1 may act as a positive regulator during the ABA-mediated inhibition of LR development (Yin et al. 2011).

Moreover, many genes function in other physiological pathways involved in LR development in rice. SHORT LATERL ROOT LENGTH 1 (SLL1), encoding a stearoylacyl carrier protein from the fatty acid desaturase family, affects overall fatty acid desaturation and also functions as a positive regulator of LR growth (Shelley et al. 2013). A pivotal factor in DNA replication, ORIGIN RECOGNITION COMPLEX SUBUNIT 3 (OsORC3), is also essential for LR development, as the Osorc3 mutant exhibited a lateral rootless phenotype in a temperaturedependent manner (Chen et al. 2013).

\section{Similarities and Differences in the Molecular Mechanisms of Crown Root and Lateral Root Development in Rice}

The developmental processes of crown roots and LRs are both tightly controlled by endogenous genetic programs that determine cell fate acquisition, cell division, and root primordia initiation, emergence, and elongation. Studies of rice mutants have led to the identification of many genes involved in LR and crown root development (Additional file 1: Table S1), some of which are involved in both processes while others are specifically involved one or the other. OsIAA23, OsARM1, OsARM2, and CRL4/OsGNOM1 play essential roles in both LR and CR development (Chhun et al. 2003b; Kitomi et al. 2008; Liu et al. 2009; Ni et al. 2011), while OsCYP2/ OsLRT2, OsIAA11, OsIAA13, and OsLRT1 are required for LR initiation but not for the development of crown roots (Chhun et al. 2003a; Wang et al. 2006; Faiyue et al. 2010; Kitomi et al. 2012; Zhu et al. 2012; Kang et al. 2013). OsCAND1 is required for crown root emergence but does not affect LR development (Wang et al. 2011). OsCRL5 and OsWOX11 are all required for the initiation of crown root development, but have no effect on LR development (Zhao et al. 2009; Kitomi et al. 2011). ARL1/CRL1 mainly affects crown root development (Liu et al. 2005). Consistant with this, the loss-of-function mutant of the ARL1/ CRL1 ortholog in maize, rootless concerning crown and seminal root (rtcs), exhibited defective crown root and embryonic seminal root initiation, but the development of the primary and lateral roots were not affected (Taramino et al. 2007). Notably, both the promoter regions of RTCS and its duplicated homologous gene RTCS-LIKE (RTCL) contain an auxin responsive element (ARE), suggesting that they are responsive to auxin just like ARL1/CRL1 (Taramino et al. 2007). It suggests that ARL1/CRL1 and its orthologs have conserved functions in crown root initiation and development in monocot cereals. LBD16 and LBD29, the two most closely related homologs of ARL1/CRL1 in Arabidopsis, are direct targets of ARF7 and ARF19 and positively regulate LR formation (Okushima et al. 2007). This observation suggests that the LBD proteins might play different roles in the dicot Arabidopsis and the monocots rice and maize. Furthermore, recent studies have indicated that rice CRL1 could positively regulate 277 genes, including key genes in meristem patterning, cell proliferation, hormone homeostasis, and LR formation (Coudert et al. 2011, 2015), suggesting that the crown root and lateral root may share some regulatory pathway.

In rice, the Auxin-OsIAA11/OsIAA13 module functions as an important negative regulator of LR formation. A similar module also exists in Arabidopsis. A dominant negative mutant of SLR/IAA14, slr1, showed no lateral root initiation (Fukaki et al. 2005, 2010). IAA14 interacts with ARF7 and ARF19 and negatively regulates LR formation (Fukaki et al. 2005; Vernoux et al. 2011), indicating that auxin stimulates lateral root initiation through the SLR/IAA14-ARF7/ARF19 signalling module. Consistently, a second auxin-signalling module involving IAA12 and ARF5 was also shown to control lateral root initiation together with SLR-ARF7/ ARF19 (De Smet et al. 2007). These data suggest a conserved function of Auxin-IAA-ARF in promoting LR formation in Arabidopsis and rice.

\section{Genes Controlling Root Hair Development}

Root hairs are long tubular outgrowths that form on the surface of specialized epidermal cells. They are required for the uptake of nutrients and water, particularly in upland conditions. Root hair development can be divided into three phases: cell specification, initiation, and elongation (Cavell and Grierson 2000). The outgrowth of root hairs is strictly regulated by genetic and environmental factors. The first root-hairless mutant reported in rice was rh2 (Suzuki et al. 2003). An exogenous application of NAA could induce very short root hairs in $r h 2$, suggesting that the absence of root hairs in this mutant may be due to a shortage of endogenous auxin; however, the gene has not yet been identified. OsWOX3A was reported to control root hair formation through the regulation of auxin transport genes (Yoo et al. 2013), further suggesting that auxin is required for root hair elongation. Root hairs are initiated normally in the Oscellulose synthase-like d1 (Oscsld1) mutant; however, this gene, which is expressed only in root hair cells, is required for their elongation (Kim et al. 2007; You et al. 2011). OsRHL1, a bHLH transcription factor expressed specifically in root hair cells, also regulates root hair elongation in rice; the loss-of-function mutants Osroot hairless 1-1 (Osrhl1-1) and Osrhl1-2 produced very short 
root hairs without affecting root length or the number of LRs and adventitious roots, suggesting that OsRHL1 functions specifically in root hair elongation (Ding et al. 2009). Furthermore, OsFORMIN HOMOLOGY 1 (OsFH1) was also found to regulate rice root hair elongation; the lossof-function mutant osfh1 exhibited root hair defects when grown submerged in solution, but produced normal root hairs in contact with the air. This root hair phenotype could not be rescued by an external supply of hormones or carbohydrates (Huang et al. 2013a). It is well known that root hair growth requires extensive cell wall modification, and recent research revealed that OsEXPA17, encoding an expansin involved in cell wall remodelling, plays a crucial role in root hair elongation. OsEXPA17 is exclusively expressed in root hair cells, and its null mutant forms short root hairs (Yu et al. 2011). Another rice gene, OsSEC14-NODULIN DOMAIN-CONTAINING PROTEIN 1 (OsSNDP1), encoding a phosphatidylinositol transfer protein (PITP), promotes root hair elongation via phospholipid signalling and metabolism, suggesting that the mediation of these processes by PITP is required for root hair elongation in rice (Huang et al. 2013b). Additionally, transgenic rice overexpressing STRESS/ABAACTIVATED PROTEIN KINASE 10 (SAPK10) produced longer root hairs, while plants overexpressing OsABILIKE 2 (OsABIL2) had attenuated ABA signalling and shorter root hairs (Wang et al. 2017), suggesting that ABA is also responsible for root hair elongation.

Up to now, almost all of the genes reported to be involved in root hair development regulate their elongation; thus, the genes regulating root hair cell specification and initiation in rice are yet to be identified.

\section{Conclusion and Discussion}

Faster and more extensive root growth is important for plant survival in complex soil conditions (de Dorlodot et al. 2007), as larger root systems typically enable plants to extract more water and nutrients from the soil (King et al. 2003). Root growth angle is also an important trait that affects rice RSA and the associated nutrient and water uptake. DEEPER ROOTING 1 (DRO1) is believed to regulate RSA by regulating the growth angle of the crown roots to adapt to drought conditions; higher levels of DRO1 expression result in deeper rooting that may maintain higher yields under drought conditions (Uga et al. 2013). LARGE ROOT ANGLE 1 (LRA1), encoding OsPIN2, was also recently identified to regulate root growth angle in rice; the lral mutant displays a shallow root system, which may benefit nutrient uptake (Wang et al. 2018). A more thorough understanding of the key genes involved in RSA and their regulation should enable breeders to breed cultivars with enhanced root systems using marker-assisted selection in the future.
Significant progress has been made in our understanding of the genetic control of root development in rice, particularly through the use of mutants with specific defects in root development and through the identification of QTLs using genetic linkage analyses. The identification of genes that regulate root traits will pave the way for more detailed genetic and molecular analyses of root system development in rice and other cereals. Though many genes that function in root development have been identified (Fig. 1; Additional file 1: Table S1), our knowledge about the molecular mechanisms of root elongation, crown root development, LR development, and root hair formation is still fragmented. The majority of studies have focussed mainly on identifying individual genes using mutants or QTL analyses, which limits our systemic understanding of the mechanisms of root development. With the rapid development of molecular biological approaches, the combination of functional genomics, transcriptomics, proteomics, and phenomics will rapidly expand our understanding of the molecular mechanisms controlling RSA.

Though many genes with key roles in RSA have been identified, little is known about the genetic improvement of the root system using these genes; can they be used in breeding? Cloning genes from QTL analyses is one feasible way to identify putative candidates for use in molecular breeding; however, it is difficult to identify QTLs with minor effects. DRO1 and PSTOL1 were both cloned from QTL analyses, and have been successfully used in breeding to improve root systems for drought tolerance and improve low-Pi tolerance for growth in less fertile soils (Gamuyao et al. 2012; Uga et al. 2013). Genome-wide association studies (GWAS) have been demonstrated to be a feasible and practical method to explore the alleles of existing varieties or genetic resources, which can then be applied in breeding. Recent studies using these approaches have identified several major QTLs containing promising candidate genes for root formation and development (Courtois et al. 2013; Biscarini et al. 2016; Bettembourg et al. 2017). However, both QTL analysis and GWAS have two main difficulties in cloning the genes associated with root development (Mai et al. 2014). First, it is difficult to do root phenotyping in real soil conditions, and the root phenotypes obtained from hydroponic and gel/agar systems do not really reflect their growth in soil. The second difficulty is the lack of precision in localizing QTLs using mapping populations or, to a much lesser extent, with association panels, because the root phenotype is more prone to vary in different growth conditions compared with the aboveground traits. Applying new approaches such as X-ray micro-computed tomography and magnetic resonance imaging to study the root system architecture in natural soils and in complex environments would enable more reliable measurement of root traits and the identification of related genes. 


\section{Additional File}

Additional file 1: Table S1. Identified genes that control root growth and development in rice. (DOC $122 \mathrm{~kb}$ )

\section{Abbreviations}

ABA: Abscisic acid; ABIL2: ABA insensitive like 2; AFB2: Auxin signaling Fbox 2; ALF: Altered lateral root formation; ARE: Auxin response element; ARF: Auxin response factor; ARL1: Adventitious rootless 1; ARM: Auxin resistant mutant; AUX/IAA: Auxin/Indole-3-Acetic acid; bHLH: Basic helixloop-helix; BRs: Brassinosteroids; CAND1: Cullin-associated and neddylationdissociated 1; CHR4: Chromatin remodelling 4; CK: Cytokinin; CKX4: CK oxidase/dehydrogenase 4; CRL: Crown rootless; CSLD1: Cellulose synthaselike D1; CYP2: Cyclophilin 2; DGL1: Dolichyl diphosphooligosaccharideprotein glycosyltransferase 1; DRO1: Deeper rooting 1; DSR-1: Drought stress response-1; EL5: Elicitor 5; ERF2: Ethylene responsive factor 2; EXPA: Expansion; FH1: Formin homology 1; GLU3: Membrane-bound endo1,4- $\beta$-glucanase; GNOM1: Membrane-associated guanine-nucleotide exchange factor of the ADP-ribosylation factor G protein (ARF-GEF); HOX1: Heme oxygenase 1; JA: Jasmonic acid; LRA1: Large root angle 1; LRs: Lateral roots; LRT: Lateral rootless; MOGS: Mannosyl-oligosaccharide glucosidase; NAA: Naphthylacetic acid; NAL: Narrow leaf; ORC3: Origin recognition complex subunit 3; PIN: Pin-formed auxin efflux carrier proteins; PSTOL1: Phosphorus-starvation tolerance 1; QC: Quiescent center; QTL: Quantitative trait loci; RAM: Root apical meristem; RHL: Root hairless; ROS: Reactive oxygen species; RPK1: Receptor protein kinase 1;

RR: Response regulator; RSA: Root system architecture; SAPK10: Stress/ABAactivated protein kinase 10; SL: Strigolactones; SLL1: Short later root length 1; SNDP1: Sec14-nodulin domain-containing protein; TIR1: Transport inhibitor response 1; TOP1: Topoisomerase 1; WOX: Wushel-related homeobox; WT: Wild type

\section{Acknowledgements}

Not applicable

\section{Funding}

This work was supported by the National Key Research and Developmen Program of China (2016YFD0100700), the National Basic Research and Development Program of China (2015CB942900), the National Natural Science Foundation of China $(31372120,31572187)$, and the Natural Science Foundation of Zhejiang province, China (LZ17C020001).

\section{Availability of Data and Materials}

Not Applicable

\section{Authors' Contributions}

The manuscript was written by FM, DX, JZ, YL, revised by CM. All the authors read and approved the final manuscript.

\section{Ethics Approval and Consent to Participate}

Not Applicable

\section{Consent for Publication}

Not Applicable

\section{Competing Interests}

The authors declare that they have no competing interests.

\section{Publisher's Note}

Springer Nature remains neutral with regard to jurisdictional claims in published maps and institutional affiliations.

Received: 14 February 2018 Accepted: 27 December 2018

Published online: 10 January 2019

\section{References}

Arite T, Kameoka H, Kyozuka J (2012) Strigolactone positively controls crown root elongation in rice. J Plant Growth Regul 31:165-172
Atkinson JA, Rasmussen A, Traini A, Voß U, Sturrock C, Mooney SJ, Wells DM, Bennett MJ (2014) Branching out in roots: uncovering form, function, and regulation. Plant Physiol 166:538-550

Benkova E, Bielach A (2010) Lateral root organogenesis-from cell to organ. Curr Opin Plant Biol 13:677-683

Bettembourg M, Dardou A, Audebert A, Thomas E, Frouin J, Guiderdoni E, Ahmadi N, Perin C, Dievart A, Courtois B (2017) Genome-wide association mapping for root cone angle in rice. Rice 10:45

Bian HW, Xie YK, Guo F, Han N, Ma S, Zeng Z, Wang J, Yang Y, Zhu M (2012) Distinctive expression patterns and roles of the miRNA393/TIR1 homolog module in regulating flag leaf inclination and primary and crown root growth in rice. (Oryza sativa L.). New Phytol 196:149-161

Biscarini F, Cozzi P, Casella L, Paolo Riccardi R, Vattari A, Orasen G, Perrini R, Tacconi G, Tondelli A, Biselli C, Cattivelli L, Spindel J, McCouch S, Abbruscato P, Valé G, Piffanelli P, Greco R (2016) Genome-wide association study for traits related to plant and grain morphology, and root architecture in temperate rice accessions. PLoS One 11:e0155425

Cavell A, Grierson CS (2000) Genetics of root hair development. In: Ridge RW Emons AMC (eds) Root hairs. Springer, Tokyo, pp 211-221

Chen X, Shi J, Hao X, Liu H, Shi J, Wu Y, Wu Z, Chen M, Wu P, Mao C (2013) OsORC3 is required for lateral root development in rice. Plant I 74:339-350

Chen Y, Yang Q, Sang S, Wei Z, Wang P (2017) Rice inositol polyphosphate kinase (OsIPK2) directly interacts with OsIAA11 to regulate lateral root formation. Plant Cell Physiol 58:1891

Chen YH, Chen YY, Shu YY, Hong CY, Kao CH (2012) Heme oxygenase is involved in nitric oxide-and auxin-induced lateral root formation in rice. Plant Cell Rep 31:1085-1091

Chhun T, Taketa S, Tsurumi S, Ichii M (2003a) The effects of auxin on lateral root initiation and root gravitropism in a lateral rootless mutant Irt1 of rice (Oryza sativa L.). Plant Growth Regul 39:161-170

Chhun T, Taketa S, Tsurumi S, Ichii M (2003b) Interaction between two auxinresistant mutants and their effects on lateral root formation in rice (Oryza sativa L.). J Exp Bot 54:2701-2708

Cho SH, Yoo SC, Zhang H, Lim JH, Paek NC (2014) Rice narrow leaf1 regulates leaf and adventitious root development. Plant Mol Biol Rep 32:270-281

Cho SH, Yoo SC, Zhang H, Pandeya D, Koh HJ, Hwang JY, Kim GT, Paek NC (2013) The rice narrow leaf2 and narrow leaf3 loci encode WUSCHEL-related homeobox 3A (OsWOX3A) and function in leaf, spikelet, tiller and lateral root development. New Phytol 198:1071-1084

Coudert Y, Bès M, Le TV, Pré M, Guiderdoni E, Gantet $P$ (2011) Transcript profiling of crown rootless 1 mutant stem base reveals new elements associated with crown root development in rice. BMC Genomics 12:1-12

Coudert Y, Le VA, Adam H, Bes M, Vignols F, Jouannic S, Guiderdoni E, Gantet P (2015) Identification of CROWN ROOTLESS1-regulated genes in rice reveals specific and conserved elements of postembryonic root formation. New Phytol 206:243-254

Coudert Y, Perin C, Courtois B, Khong NG, Gantet P (2010) Genetic control of root development in rice, the model cereal. Trends Plant Sci 15:219-226

Courtois B, Audebert A, Dardou A, Roques S, Ghneim-Herrera T, Droc G, Frouin J, Rouan L, Gozé E, Kilian A, Ahmadi N, Dingkuhn M (2013) Genome-wide association mapping of root traits in a japonica rice panel. PLoS One 8:e78037

Cui P, Liu H, Ruan S, Ali B, Gill RA, Ma H, Zheng Z, Zhou W (2017) A zinc finger protein, interacted with cyclophilin, affects root development via IAA pathway in rice. J Integr Plant Biol 59:496-505

de Dorlodot S, Forster B, Pagès L, Price A, Tuberosa R, Draye X (2007) Root system architecture: opportunities and constraints for genetic improvement of crops. Trends Plant Sci 12:474-481

De Smet I, Tetsumura T, De Rybel B, Frei dit Frey N, Laplaze L, Casimiro I, Swarup $R$, Naudts M, Vanneste S, Audenaert D, Inzé D, Bennett MJ, Beeckman T (2007) Auxin-dependent regulation of lateral root positioning in the basal meristem of Arabidopsis. Development 134:681

Debi BR, Mushika J, Taketa S, Miyao A, Hirochika H, Ichii M (2003) Isolation and characterization of a short lateral root mutant in rice (Oryza sativa L.). Plant Sci 165:895-903

Ding W, Yu Z, Tong Y, Huang W, Chen H, Wu P (2009) A transcription factor with a bHLH domain regulates root hair development in rice. Cell Res 19:1309-1311

Faiyue B, Vijayalakshmi C, Nawaz S, Nagato Y, Taketa S, Ichii M, Al-Azzawi MJ, Flowers TJ (2010) Studies on sodium bypass flow in lateral rootless mutants Irt1 and Irt2, and crown rootless mutant crl1 of rice (Oryza sativa L.). Plant Cell Environ 33:687-701

Fukai S, Cooper M (1995) Development of drought resistant cultivars using physio-morphological traits in rice. Field Crops Res 40:67-87 
Fukaki H, Nakao Y, Okushima Y, Theologis A, Tasaka M (2005) Tissue-specific expression of stabilized SOLITARY-ROOT/IAA14 alters lateral root development in Arabidopsis. Plant J 44:382-395

Fukaki H, Tameda S, Masuda H, Tasaka M (2010) Lateral root formation is blocked by a gain-of function mutation in the SOLITARY-ROOT/IAA14 gene of Arabidopsis. Plant J 29:153-168

Gamuyao R, Chin JH, Pariasca-Tanaka J, Pesaresi P, Catausan S, Dalid C, SlametLoedin I, Tecson-Mendoza EM, Wissuwa M, Heuer S (2012) The protein kinase PSTOL1 from traditional rice confers tolerance of phosphorus deficiency. Nature 488:535-539

Gao S, Fang J, Xu F, Wang W, Sun X, Chu J, Cai B, Feng Y, Chu C (2014) Cytokinin oxidase/dehydrogenase 4 integrates cytokinin and auxin signaling to control rice crown root formation. Plant Physiol 165:1035-1046

Gojon A, Nacry P, Davidian JC (2009) Root uptake regulation: a central process for NPS homeostasis in plants. Curr Opin Plant Biol 12:328-338

Gowda VRP, Henry A, Yamauchi A, Shashidha HE, Serraj R (2011) Root biology and genetic improvement for drought avoidance in rice. Field Crop Res 122:1-13

Gu M, Zhang J, Li H, Meng D, Li R, Dai X, Wang S, Liu W, Qu H, Xu G (2017) Maintenance of phosphate homeostasis and root development are coordinately regulated by MYB1, an R2R3-type MYB transcription factor in rice. J Exp Bot 68:3603-3615

Hirose N, Makita N, Kojima M, Kamada-Nobusada T, Sakakibara H (2007) Overexpression of a type-a response regulator alters rice morphology and cytokinin metabolism. Plant Cell Physiol 48:523-539

Hsu YY, Chao YY, Kao CH (2012) Methyl jasmonate-induced lateral root formation in rice: the role of heme oxygenase and calcium. J Plant Physiol 170:63-69

Huang J, Kim CM, Xuan YH, Liu J, Kim TH, Kim BK, Han CD (2013a) Formin homology $1(\mathrm{OsFH} 1)$ regulates root-hair elongation in rice (Oryza sativa L.). Planta 237:1227-1239

Huang J, Kim CM, Xuan YH, Park SJ, Piao HL, Je Bl, Liu J, Kim TH, Kim BK, Han CD (2013b) OsSNDP1, a Sec14-nodulin domain-containing protein, plays a critical role in root hair elongation in rice. Plant Mol Biol 82:39-50

Inukai Y, Sakamoto T, Ueguchi-Tanaka M, Shibata Y, Gomi K, Umemura I, Hasegawa Y, Ashikari M, Kitano H, Matsuoka M (2005) CROWN ROOTLESS1, which is essential for crown root formation in rice, is a target of an AUXIN RESPONSE FACTOR in auxin signaling. Plant Cell 17:1387-1396

Itoh J, Nonomura K, Ikeda K, Yamaki S, Inukai Y, Yamagishi H, Kitano H, Nagato $Y$ (2005) Rice plant development: from zygote to spikelet. Plant Cell Physiol 46:23-47

Jia L, Wu Z, Hao X, Carrie C, Zheng L, Whelan J, Wu Y, Wang S, Wu P, Mao C (2011) Identification of a novel mitochondrial protein, SHORT POSTEMBRYONIC ROOTS 1 (SPR1), involved in root development and iron homeostasis in Oryza sativa. New Phytol 189:843-855

Jia L, Zhang B, Mao C, Li J, Wu Y, Wu P, Wu Z (2008) OsCYT-INV1 for alkaline/ neutral invertase is involved in root cell development and reproductivity in rice (Oryza sativa L.). Planta 228:51-59

Jiang H, Wang S, Dang L, Wang S, Chen H, Wu Y, Jiang X, Wu P (2005) A novel short-root gene encodes a glucosamine-6-phosphate acetyltransferase required for maintaining normal root cell shape in rice. Plant Physiol 138: 232-242

Jing H, Yang X, Zhang J, Liu X, Zheng H, Dong G, Nian J, Feng J, Xia B, Qian Q, Li J, Zuo J (2015) Peptidyl-prolyl isomerization targets rice aux/IAAs for proteasomal degradation during auxin signaling. Nat Commun 6:7395

Kang B, Zhang Z, Wang L, Zheng L, Mao W, Li M, Wu Y, Wu P, Mo X (2013) OSCYP2, a chaperone involved in degradation of auxin-responsive proteins, plays crucial roles in rice lateral root initiation. Plant J 74:86-97

Kim CM, Park SH, Je Bl, Park SH, Park SJ, Piao HL, Eun MY, Dolan L, Han CD (2007) OsCSLD1, a cellulose synthase-like D1 gene, is required for root hair morphogenesis in rice. Plant Physiol 143:1220-1230

King J, Gay A, Sylvester-Bradley R, Bingham I, Foulkes J, Gregory P, Robinson D (2003) Modelling cereal root systems for water and nitrogen capture: towards an economic optimum. Ann Bot 91:383-390

Kitomi Y, Inahashi H, Takehisa H, Sato Y, Inukai Y (2012) OsIAA13-mediated auxin signaling is involved in lateral root initiation in rice. Plant Sci 190:116-122

Kitomi $Y$, Ito $H$, Hobo T, Aya K, Kitano H, Inukai $Y$ (2011) The auxin responsive AP2/ERF transcription factor CROWNROOTLESS5 is involved in crown root initiation in rice through the induction of OsRR1, a type-a response regulator of cytokinin signaling. Plant J 67:472-484

Kitomi Y, Ogawa A, Kitano H, Inukai Y (2008) CRL4 regulates crown root formation through auxin transport in rice. Plant Root 2:19-28
Koiwai H, Tagiri A, Katoh S, Katoh E, Ichikawa H, Minami E, Nishizawa Y (2007) $\mathrm{RING}-\mathrm{H} 2$ type ubiquitin ligase EL5 is involved in root development through the maintenance of cell viability in rice. Plant J 51:92-104

Li J, Han Y, Liu L, Chen Y, Du Y, Zhang J, Sun H, Zhao Q (2015a) Qrt9, a quantitative trait locus controlling root thickness and root length in upland rice. J Exp Bot 66:2723-2732

Li J, Zhao Y, Chu H, Wang L, Fu Y, Liu P, Upadhyaya N, Chen C, Mou T, Feng Y, Kumar P, Xu J (2015b) SHOEBOX modulates root meristem size in rice through dose-dependent effects of gibberellins on cell elongation and proliferation. PLOS Genet 11:e1005464

Li J, Zhu S, Song X, Shen Y, Chen H, Yu J, Yi K, Liu Y, Karplus VJ, Wu P, Deng XW (2006) A rice glutamate receptor-like gene is critical for the division and survival of individual cells in the root apical meristem. Plant Cell 18:340-349

Liu H, Wang S, Yu X, Yu J, He X, Zhang S, Shou H, Wu P (2005) ARL1, a LOBdomain protein required for adventitious root formation in rice. Plant $J$ 43:47-56

Liu S, Wang J, Wang L, Wang X, Xue Y, Wu P, Shou H (2009) Adventitious root formation in rice requires OsGNOM1 and is mediated by the OsPINs family. Cell Res 19:1110-1119

Mai CD, Phung NTP, To H M, Mathieu Gonin M, Hoang GT, Nguyen KL, Do VN, Courtois B, Gantet P (2014) Genes controlling root development in rice. Rice 7:30

Mao C, Wang S, Jia Q, Wu P (2006) OsEIL1, a rice homolog of the Arabidopsis EIN3 regulates the ethylene response as a positive component. Plant $\mathrm{Mol}$ Biol 61:141-152

Mori M, Nomura T, Ooka H, Ishizaka M, Yokota T, Sugimoto K, Okabe K, Kajiwara H, Satoh K, Yamamoto K, Hirochika H, Kikuchi S (2002) Isolation and characterization of a rice dwarf mutant with a defect in brassinosteroid biosynthesis. Plant Physiol 130:1152-1161

Ni J, Wang G, Zhu Z, Zhang H, Wu Y, Wu P (2011) OsIAA23-mediated auxin signaling defines postembryonic maintenance of QC in rice. Plant J 68: 433-442

Obara M, Tamura W, Ebitani T et al (2010) Fine-mapping of qRL6.1, a major QTL for root length of rice seedlings grown under a wide range of $\mathrm{NH}_{4}+$, concentrations in hydroponic conditions. Theor Appl Genet 121:535-547

Okushima Y, Fukaki H, Onoda M, Theologis A, Tasaka M (2007) ARF7 and ARF19 regulate lateral root formation via direct activation of $L B D / A S L$ genes in Arabidopsis. Plant Cell 19:118-130

Qi Y, Wang S, Shen C, Zhang S, Chen Y, Xu Y, Liu Y, Wu Y, Jiang D (2012) OsARF12, a transcription activator on auxin response gene, regulates root elongation and affects iron accumulation in rice (Oryza sativa L.). New Phytol 193:109-120

Qin C, Cheng L, Zhang H, He M, Shen J, Zhang Y, Wu P (2016) OsGatb, the subunit of tRNA-dependent amidotransferase, is required for primary root development in rice. Front Plant Sci 7:599-605

Qin C, Li Y, Gan J, Wang W, Zhang H, Liu Y, Wu P (2013) OsDGL1, a homolog of an oligosaccharyltransferase complex subunit, is involved in $\mathrm{N}$-glycosylation and root development in rice. Plant Cell Physiol 54:129-137

Shafiq S, Chen C, Yang J, Cheng L, Ma F, Widemann E, Sun Q (2017) DNA Topoisomerase 1 prevents R-loop accumulation to modulate auxin-regulated root development in rice. Mol Plant 10:821-833

Shelley IJ, Nishiuchi S, Shibata K, Inukai Y (2013) SLL1, which encodes a member of the stearoyl-acyl carrier protein fatty acid desaturase family, is involved in cell elongation in lateral roots via regulation of fatty acid content in rice. Plant Sci 207:12-17

Sun H, Bi Y, Tao J, Huang S, Hou M, Xue R, Liang Z, Gu P, Yoneyama K, Xie X, Shen Q, Xu G, Zhang Y (2016) Strigolactones are required for nitric oxide to induce root elongation in response to nitrogen and phosphate deficiencies in rice. Plant Cell Environ 39:1473-1484

Sun H, Tao J, Hou M, Huang S, Chen S, Liang Z, Xie T, Wei Y, Xie X, Yoneyama K, Xu G, Zhang Y (2015) A strigolactone signal is required for adventitious root formation in rice. Ann Bot 115:1155-1162

Suzuki N, Taketa S, Ichii M (2003) Morphological and physiological characteristics of a root-hairless mutant in rice (Oryza sativa L.). Plant Soil 255:9-17

Taramino G, Sauer M, Stauffer JL Jr, Multani D, Niu X, Sakai H, Hochholdinger F (2007) The maize (Zea mays L.) RTCS gene encodes a LOB domain protein that is a key regulator of embryonic seminal and post-embryonic shootborne root initiation. Plant J 50:649-659

Toorchi M, Shashidhar HE, Sharma N, Hittalmani S (2002) Tagging QTLs for maximum root length in rain fed lowland rice (Oryza sativa L.) using molecular markers. Cell Mol Biol Lett 7:771-776 
Toorchi M, Shashidhar HE, Sharma N, Hittalmani S (2007) Tagging QTLs for maximum root length in rain fed lowland rice by combined selective genotyping and STMs markers. J Food Agric Environ 5:209-210

Uga Y, Sugimoto K, Ogawa S, Rane J, Ishitani M, Hara N, Kitomi Y, Inukai Y, Ono K, Kanno N, Inoue H, Takehisa H, Motoyama R, Nagamura Y, Wu J, Matsumoto T, Takai T, Okuno K, Yano M (2013) Control of root system architecture by DEEPER ROOTING 1 increases rice yield under drought conditions. Nat Genet 45:1097-1102

Vernoux T, Brunoud G, Farcot E et al (2011) The auxin signaling network translates dynamic input into robust patterning at the shoot apex. Mol Syst Biol 7:508

Wang H, Taketaa S, Miyaob A, Hirochikab H, Ichiia M (2006) Isolation of a novel lateral-rootless mutant in rice (Oryza sativa L.) with reduced sensitivity to auxin. Plant Sci 170:70-77

Wang H, Xu X, Zhan X, Zhai R, Wu W, Shen X, Dai G, Cao L, Cheng S (2013) Identification of qRL7, a major quantitative trait locus associated with rice root length in hydroponic conditions. Breed Sci 63:267-274

Wang L, Guo M, Li Y, Ruan W, Mo X, Wu Z, Sturrock CJ, Yu H, Lu C, Peng J, Mao C (2018) LARGE ROOT ANGLE1, encoding OsPIN2, is involved in root system architecture in rice. J Exp Bot 69:385-397

Wang S, Xu Y, Li Z, Zhang S, Lim JM, Lee KO, Li C, Qian Q, Jiang A, Qi Y (2014a) OsMOGS is required for $\mathrm{N}$-glycan formation and auxin-mediated root development in rice (Oryza sativa L.). Plant J 78:632-645

Wang T, Li C, Wu Z, Jia Y, Wang H, Sun S, Mao C, Wang X (2017) Abscisic acid regulates auxin homeostasis in rice root tips to promote root hair elongation. Front Plant Sci 8:1121-1138

Wang XF, He FF, Ma XX, Mao CZ, Hodgman C, Lu CG, Wu P (2011) OsCAND1 is required for crown root emergence in rice. Mol Plant 4:289-299

Wang Y, Ma N, Qiu S, Zou H, Zang G, Kang Z, Wang G, Huang J (2014b) Regulation of the a-expansin gene OsEXPA8 expression affects root system architecture in transgenic rice plants. Mol Breed 34:47-57

Wang Y, Wang D, Gan T, Liu L, Long W, Wang Y, Niu M, Li X, Zheng M, Jiang L, Wan J (2016) CRL6, a member of the CHD protein family, is required for crown root development in rice. Plant Physiol Bioch 105:185-194

Xia J, Yamaji N, Che J, Shen RF, Ma JF (2014) Normal root elongation requires arginine produced by argininosuccinate lyase in rice. Plant J 78:215-226

Xiao G, Qin H, Zhou J, Quan R, Lu X, Huang R, Zhang H (2016) OsERF2 controls rice root growth and hormone responses through tuning expression of key genes involved in hormone signaling and sucrose metabolism. Plant Mol Biol 90:293-302

Xu L, Zhao H, Ruan W, Deng M, Wang F, Peng J, Luo J, Chen Z, Yi K (2017) ABNORMAL INFLORESCENCE MERISTEM1 functions in salicylic acid biosynthesis to maintain proper reactive oxygen species levels for root MERISTEM activity in rice. Plant Cell 29:560-574

Xu M, Zhu L, Shou H, Wu P et al (2005) A PIN1 family gene, OsPIN1, involved in auxin-dependent adventitious root emergence and tillering in rice. Plant Cell Physiol 46:1674-1681

Yamamoto Y, Kamiya N, Morinaka Y, Matsuoka M, Sazuka T (2007) Auxin biosynthesis by the YUCCA genes in rice. Plant Physiol 143:1362-1371

Yin XM, Rocha PSCF, Wang ML, Zhu YX, Li LY, Song SF, Xia X (2011) Rice gene OsDSR-1, promotes lateral root development in Arabidopsis, under highpotassium conditions. J Plant Biol 54:180-189

Yoo SC, Cho SH, Paek NC (2013) Rice WUSCHEL-related homeobox 3A (OsWOX3A) modulates auxin-transport gene expression in lateral root and root hair development. Plant Signal Behav 8:e25929

Yoshida K, Imaizumi N, Kaneko S, Kawagoe Y, Tagiri A, Tanaka H, Nishitani K, Komae K (2006) Carbohydrate-binding module of a rice endo- $\beta-1,4-$ glycanase, OsCel9A, expressed in auxin-induced lateral root primordia, is post-translationally truncated. Plant Cell Physiol 47:1555-1571

You T, Shiotani K, Shitsukawa N, Miyao A, Hirochika H, Ichii M, Taketa S (2011) Root hairless 2 (rth2) mutant represents a loss-of-function allele of the cellulose synthase-like gene OsCSLD1 in rice (Oryza sativa L.). Breed Sci 61:225-233

Yu C, Sun C, Shen C, Wang S, Liu F, Liu Y, Chen Y, Li C, Qian Q, Aryal B, Geisler M, Jiang de A, Qi Y (2015) The auxin transporter, OsAUX1, is involved in primary root and root hair elongation and in cd stress responses in rice (Oryza sativa L.). Plant J 83:818-830

Yu Z, Kang B, He X, Lv S, Bai Y, Ding W, Chen M, Cho HT, Wu P (2011) Root hairspecific expansins modulate root hair elongation in rice. Plant J 66:725-734

Zhang J, Xu L, Wu Y, Chen X, Liu Y, Zhu S, Ding W, Wu P, Yi K (2012) OsGLU3, a putative membrane-bound endo-1,4-beta-glucanase, is required for root cell elongation and division in rice (Oryza sativa L.). Mol Plant 5:176-186
Zhang WP, Shen XY, Wu P, Hu B, Liao CY (2001) QTLs and epistasis for seminal root length under a different water supply in rice (Oryza sativa L.). Theor Appl Genet 103:118-123

Zhao C, Xu J, Chen Y, Mao C, Zhang S, Bai Y, Jiang D, Wu P (2012) Molecular cloning and characterization of OsCHR4, a rice chromatin-remodeling factor required for early chloroplast development in adaxial mesophyll. Planta 236:1165-1176

Zhao H, Ma T, Wang X, Deng Y, Ma H, Zhang R, Zhao J (2015a) OsAUX1 controls lateral root initiation in rice (Oryza sativa L.). Plant Cell Environ 38:2208-2222

Zhao Y, Cheng S, Song Y, Huang Y, Zhou S, Liu X, Zhou DX (2015b) The interaction between rice ERF3 and WOX11 promotes crown root development by regulating gene expression involved in cytokinin signaling. Plant Cell 27:2469-2483

Zhao Y, Hu Y, Dai M, Huang L, Zhou DX (2009) The WUSCHEL-related homeobox gene WOX11 is required to activate shoot-borne crown root development in rice. Plant Cell 21:736-748

Zheng H, Li S, Ren B, Zhang J, Ichii M, Taketa S, Tao Y, Zuo J, Wang H (2013) LATERAL ROOTLESS2, a cyclophilin protein, regulates lateral root initiation and auxin signaling pathway in rice. Mol Plant 6:1719-1721

Zhou S, Jiang W, Long F, Cheng S, Yang W, Zhao Y, Zhou DX (2017) Rice homeodomain protein WOX11 recruits a histone acetyltransferase complex to establish programs of cell proliferation of crown root meristem. Plant Cell 29:1088-1104

Zhu Z, Liu Y, Liu S, Mao C, Wu Y, Wu P (2012) A gain-of-function mutation in OsIAA11 affects lateral root development in rice. Mol Plant 5:154-161

Zou Y, Liu X, Wang Q, Chen Y, Liu C, Qiu Y, Zhang W (2014) OsRPK1, a novel leucine-rich repeat receptor-like kinase, negatively regulates polar auxin transport and root development in rice. Biochim Biophys Acta 1840:1676-1685

\section{Submit your manuscript to a SpringerOpen ${ }^{\circ}$ journal and benefit from:}

- Convenient online submission

- Rigorous peer review

- Open access: articles freely available online

High visibility within the field

- Retaining the copyright to your article

Submit your next manuscript at $>$ springeropen.com 